\title{
A Sparse Transmission Disequilibrium Test for Haplotypes Based on Bradley- Terry Graphs
}

\author{
Li Ma $^{a}$ Wing Hung Wong ${ }^{b, c}$ Art B. Owen ${ }^{b}$

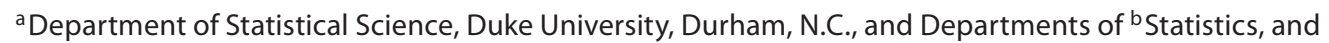 \\ 'Health Research and Policy, Stanford University, Stanford, Calif., USA
}

\section{Key Words}

Case-parent trio $\cdot$ Family-based study $\cdot$ Linkage $\cdot$ Linkage

disequilibrium $\cdot$ Penalized logistic regression

\begin{abstract}
Background: Linkage and association analysis based on haplotype transmission disequilibrium can be more informative than single marker analysis. Several works have been proposed in recent years to extend the transmission disequilibrium test (TDT) to haplotypes. Among them, a powerful approach called the evolutionary tree TDT (ET-TDT) incorporates information about the evolutionary relationship among haplotypes using the cladogram of the locus. Methods: In this work we extend this approach by taking into consideration the sparsity of causal mutations in the evolutionary history. We first introduce the notion of a Bradley-Terry (BT) graph representation of a haplotype locus. The most important property of the BT graph is that sparsity of the edge set of the graph corresponds to small number of causal mutations in the evolution of the haplotypes. We then propose a method to test the null hypothesis of no linkage and association against sparse alternatives under which a small number of edges on the BT graph have non-nil effects. Results and Conclusion: We compare the performance of our approach to that of the ET-TDT through a power study, and show that incorporating sparsity of causal mutations can significantly improve the power of a haplotype-based TDT.
\end{abstract}

Copyright $\odot 2012$ S. Karger AG, Basel

\section{KARGER}

Fax +4161306 1234

E-Mail karger@karger.ch

www.karger.com
(C) 2012 S. Karger AG, Basel

0001-5652/12/0731-0052\$38.00/0

Accessible online at:

www.karger.com/hhe

\section{Introduction}

Recent advances in sequencing methods have made it feasible to obtain sequence reads that are completely phased across entire chromosomes [1]. From these sequences one can obtain arbitrarily large haplotypes without the need for genotyping relatives. As the cost of sequencing continues its rapid decrease, experimentally determined long-range haplotype data will become available for human genetic studies. The exploitation of haplotype information may lead to new and more powerful study designs and data analysis methods. Here, we take a step in this direction by proposing a sparsely parameterized model for the transmission of haplotypes among parents and children. We will exploit the model to construct more efficient versions of the transmission disequilibrium test (TDT) [2-6] for trait-locus association.

Since its introduction, the TDT framework has become a popular method for testing linkage and association between genetic markers and disease loci. A desirable feature of this framework, in comparison to the population association study design, is its robustness to subpopulation structures. In recent years, several works [7-13] have been proposed to extend the TDT framework from the test of single markers to that of haplotypes. A major challenge such analysis encounters is the increased degrees of freedom due to the large number of haplotyp-

\section{Li Ma}

Department of Statistical Science

Duke University

Box 90251, Durham, NC 27708-0251 (USA)

Tel. +1 919684 2871, E-Mail li.ma@ duke.edu 
ic alleles. One way to deal with this difficulty is to combine haplotypes with similar disease risk. A brute-force implementation of this strategy would incur a huge number of hypothesis tests for determining which haplotypes have similar risk. Seltman et al. [10] proposed a method called evolutionary tree TDT (ET-TDT), which reduces the number of tests by utilizing the evolutionary history of the haplotypes, following the idea introduced by Templeton and colleagues in a sequence of papers [14-18]. More specifically, instead of comparing each haplotype to all other possible subsets of haplotypes at a locus, ETTDT compares a set of haplotypes to their nearest evolutionary neighbor, and merges the neighbor with that set in terms of their disease risk if the (local) null hypothesis $\left(H_{0}\right)$ - that the neighbor does not have different disease risk - is not rejected at a Bonferroni-corrected significance level.

ET-TDT utilizes the evolutionary history through the cladogram of the haplotypes. The cladogram is a graphical representation of the evolutionary relationships among the alleles. Each vertex in a cladogram represents a haplotype while each edge links two haplotypes that are evolutionary neighbors. (That is, one haplotype arises from the other through one or more mutations.) The testing strategy employed by the ET-TDT is aimed at finding those edges for which the haplotypes lying on the two sides display differential risk of disease, as reflected in the unbalanced transmission probability of one side versus the other. From now on we shall refer to this as an edge effect. This strategy is intuitive - when a causal mutation (possibly unobserved) occurs along some branch of the underlying evolutionary tree, the haplotypes that lie on the two sides of that branch will have different disease risks. (Of course, because not all mutations are observed in the data, the edges in a cladogram typically do not correspond perfectly to the branches in the full evolutionary tree.)

The current work extends the ET-TDT by taking into consideration the 'sparsity' of causal mutations. More specifically, because disease-related mutations do not occur frequently, a typical disease susceptibility locus is expected to contain only a small number of such mutations. (This does not exclude the possibility that there are a large number of different causal rare mutations across different locations on the genome.) As will be demonstrated in this work, incorporating this sparseness into inference can substantially reduce the model space and thereby improve the power for detecting association and linkage.

To appropriately account for this sparsity structure, we introduce a notion called the Bradley-Terry (BT) graph. The BT graph is not just a graphical representation of the evolutionary history of haplotypes (e.g. the cladogram), which does not relate to disease risks directly, nor is it just the BT model for haplotype transmission, as that model only deals with pair-wise transmission odds and ignores the cladogram structure. The BT graph encapsulates both features by defining a set of edge parameters on the cladogram graph in such a way that the result is a subclass of the BT model. The most important property of the BT graph is that sparsity of the edge set of the graph corresponds to small number of causal mutations in the evolution of the haplotypes.

Our proposed approach to testing haplotype transmission disequilibrium based on the BT graph can be summarized as follows. We first construct the BT graph representation for a haplotype locus and, based on this representation, impose sparsity onto the edge set by introducing a penalization term into the conditional likelihood. In particular, we adopt the Lasso $\left(L_{1}\right)$ penalty to obtain sparse estimates of the model. The amount of penalization can either be preset or be determined based on the data through cross-validation. Finally, the statistical significance of the final model so chosen versus the $H_{0}$ model can be judged using permutation testing. We carry out a power study based on simulation under a variety of disease models and compare our method to the ET-TDT.

\section{Methods}

The BT Graph Representation for a Haplotype Locus

We consider the case where the data consists of patient-parent trios. Each parent contains two haplotypes (possibly identical), and we observe what haplotype (up to uniqueness) is transmitted onto the patient (the child). Suppose at the locus under investigation there are a total of $M$ distinct haplotypes. We let $(i, j)$ denote the pair of haplotypes a parent has, where $i, j=1,2, \ldots, M$, and let $\pi_{i \mid j}$ denote the chance for haplotype $i$ to be transmitted and $j$ untransmitted. So $\pi_{i \mid j}=1-\pi_{j \mid i}$. The BT model for haplotype transmission specifies these transmission probabilities as

$$
\log \left(\pi_{i \mid j} / \pi_{j \mid i}\right)=\beta_{i}-\beta_{j},
$$

where $\beta_{i}$ is a measure of disease risk of haplotype $i$, so (conditional on the disease status of the child) haplotypes with higher disease risk are more likely to be transmitted. For identifiability, one can set $\beta_{1}=0$ as the baseline risk, leaving $M-1$ free parameters in the model. Under $H_{0}$ of no association, $\beta_{1}=\beta_{2}=\ldots=\beta_{M}$. An interesting feature of the BT model is its 'transitivity'. More specifically, for another haplotype $k$,

$$
\begin{aligned}
& \log \left(\pi_{i \mid k} / \pi_{k \mid i}\right)=\beta_{i}-\beta_{k}=\left(\beta_{i}-\beta_{j}\right)+\left(\beta_{j}-\beta_{k}\right)=\log \left(\pi_{i \mid j} / \pi_{j \mid i}\right) \\
& +\log \left(\pi_{j \mid k} / \pi_{k \mid j}\right) .
\end{aligned}
$$

This transitivity property of the BT model allows us to reparameterize the haplotype parameters $\beta \mathrm{s}$ in terms of the edge effects on the corresponding cladogram for the locus under in- 


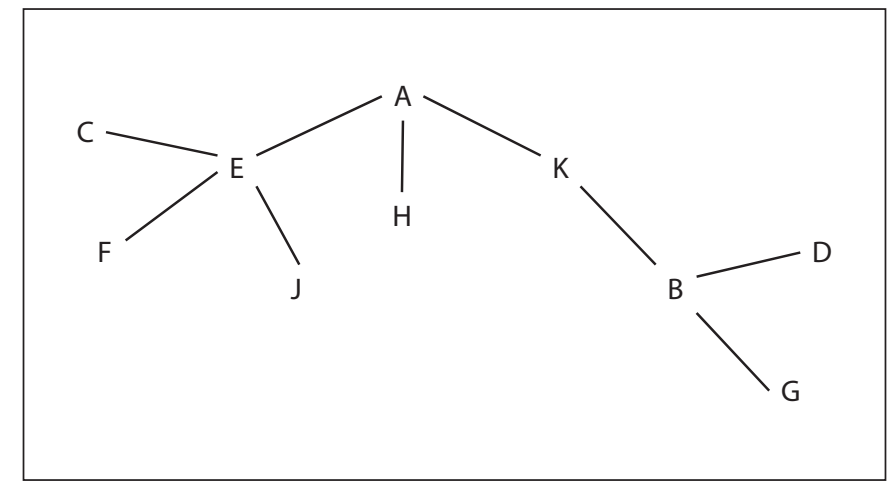

Fig. 1. Cladogram of haplotypes. This example is originally from Hallman et al. [19] and was adopted in Seltman et al. [10].

vestigation. To see this, we first let $G=\{V, E\}$ denote the cladogram of the locus under investigation, where $V=\{1,2, \ldots, M\}$ is the set of vertices (i.e. distinct haplotypes) and $E$ is the set of edges that link haplotypes that are evolutionary neighbors. That is, $E=$ $\left\{\left\{i_{1}, i_{2}\right\}\right.$ : haplotype $i_{1}$ and haplotype $i_{2}$ are evolutionary neighbors, $i_{1}$ and $\left.i_{2} \in V\right\}$.

Note that a cladogram is an undirected graph so $\left\{i_{1}, i_{2}\right\}$ and $\left\{i_{2}, i_{1}\right\}$ denote the same edge. Because a cladogram does not contain loops, there are in total of $M-1$ edges in $E$. For each $\left\{i_{1}, i_{2}\right\} \in E$, let

$$
\gamma_{i_{1}, i_{2}}=\beta_{i_{1}}-\beta_{i_{2}}
$$

denote the edge effect of $\left\{i_{1}, i_{2}\right\}$. Note that $\gamma_{i_{2}, i_{1}}=-\gamma_{i_{1}, i_{2}}$, so effectively we have $M-1$ free parameters for edge effects. We let $\gamma_{E}$ denote the collection of edge effects for all edges in $E$.

The transmission probability for $i_{1}$ versus $i_{2}$ satisfies $\log \left(\pi_{i_{1}} \mid i_{2} /\right.$ $\left.\pi_{i_{2} \mid i_{1}}\right)=\gamma_{i_{1}, i_{2}}$. More generally, for two haplotypes $i, j \in V$ that are not necessarily directly connected by a single edge, let $\left(i_{1}, i_{2}, \ldots, i_{k}\right)$ denote a path that connects $i$ to $j$. That is, $i_{1}=i$ and $i_{k}=j$ with $\left\{i_{m}\right.$, $\left.i_{m+1}\right\} \in E$ for $m=1,2, \ldots, k-1$. (Due to the acyclic nature of the cladogram, from any one haplotype to another, there exists a unique path consisting of distinct haplotypes. From now on, we use the path to refer to this particular one.) The transitivity of the BT model gives us

$$
\log \left(\pi_{i \mid j} / \pi_{j \mid i}\right)=\sum_{m=1}^{k-1} \log \left(\pi_{i_{m} \mid i_{m+1}} / \pi_{i_{m+1} \mid i_{m}}\right)=\sum_{m=1}^{k-1} \gamma_{i_{m}, i_{m+1}} .
$$

This completes the specification of the BT model in terms of the edge effects. We shall from now on refer to this representation of a haplotype locus using the cladogram $G=\{V, E\}$ and the collection of the edge effects $\gamma_{E}$ as the BT graph for the locus.

This representation can best be understood through a simple example. Seltman et al. [10] used an example of a locus consisting of five bi-allelic markers when introducing the original ET-TDT method. (This example was originally presented in Hallman et al. [19] for a study of the relation between apolipoprotein B and cholesterol.) For ease of comparison, we continue to adopt the same example. There are 10 distinct haplotypes at the locus and their definition in terms of the marker alleles are given in table 1 and the corresponding cladogram constructed by parsimony is given in figure 1 .
For this cladogram, the vertex set $V=\{A, B, C, D, E, F, G, H, J$, $K\}$, and the edge set $E=\{\{A, E\},\{A, H\},\{A, K\},\{C, E\},\{E, F\},\{E, J\}$, $\{B, K\},\{B, D\},\{B, G\}\}$. The path from, say, haplotype $D$ to $E$ is $(D$, $B, K, A, E)$. Thus under the $\mathrm{BT}$ graph representation of the locus, the $\log$ odds for transmission of $D$ versus $E$ is

$$
\begin{aligned}
\log \left(\pi_{D \mid E} / \pi_{E \mid D}\right) & =\beta_{D}-\beta_{E}=\gamma_{D, B}+\gamma_{B, K}+\gamma_{K, A}+\gamma_{A, E} \\
& =-\gamma_{B, D}+\gamma_{B, K}-\gamma_{A, K}+\gamma_{A, E}
\end{aligned}
$$

\section{Sparse Edge Effects in BT Graphs and $L_{1}$ Penalized} Conditional Logistic Regression

Let $l^{(C)}$ denote the conditional log-likelihood of the transmission events for all patient-parent trios given the parental haplotypes. The contribution from a parent, indexed by $x$, to $l^{(C)}$ is $\log p_{i(x) \mid j(x)}$, where $\{i(x), j(x)\}$ denotes the parent's two haplotypes with $i(x)$ being the transmitted allele. Let $\left(i_{1}(x), i_{2}(x), \ldots, i_{k(x)}(x)\right)$ be the path from $i(x)$ to $j(x)$. Then under the BT graph representation, the total log conditional likelihood over all parents can be written as

$$
l^{(C)}=-\sum_{x \in \text { Parents }} \log \left(1+\exp \left(-\sum_{h=1}^{k(x)-1} \gamma_{i_{h}(x), i_{h+1}(x)}\right)\right) .
$$

This is simply a conditional logistic regression (CLR), and the maximum likelihood estimates for the edge effects can be computed by maximizing $l^{(C)}$. A joint hypothesis test against the global $H_{0}: \gamma_{i_{1}}, i_{2}=0$ for all $\left\{i_{1}, i_{2}\right\} \in E$ can be carried out by standard methods such as a likelihood ratio test. However, because there are typically a small number of causal mutations at any susceptibility locus, even when $H_{0}$ is not true, most of the edges will have nil effects, especially for loci with a large number of haplotypes. Therefore, such a joint testing approach that incurs a degree of freedom equal to $M-1$ may not be efficient unless the proportion of non-nil edge effects is sufficiently large. (This will be demonstrated in our later power studies.)

One can thus improve the statistical power by exploiting the sparsity structure of the edge effects. To this end we introduce a Lasso $\left(L_{1}\right)$ regularization term on the edge effects into the conditional likelihood [20]. The penalized log-likelihood is

$$
l_{p}^{(C)}=-\sum_{x \in \text { Parents }} \log \left(1+\exp \left(-\sum_{h=1}^{k(x)-1} \gamma_{i_{h}(x), i_{h+1}(x)}\right)\right)-\lambda \sum_{\{l, m\} \in E}\left|\gamma_{l, m}\right|,
$$

where $\lambda$ is a tuning parameter that controls the amount of sparsity. Maximizing $l \underset{p}{(C)}$ will generate estimates of the edge effects, including one or more exact zeroes for large enough $\lambda$. The number of zero estimates is increasing or flat as $\lambda$ increases. Several strategies can be adopted in choosing $\lambda$. For example, one can choose it so that exactly a pre-specified number of edge effects will be non-zero. In the current context, however, it is preferable to choose $\lambda$ adaptively using procedures such as $k$-fold cross-validation $(k-C V)$. This allows the data to suggest the appropriate level of regularization. It also makes the method robust to potential misspecifications of the underlying cladogram. More specifically, note that when some edges are misplaced in the inferred cladogram, transitivity of the BT model will still ensure the validity of the reparameterization using edge effects. However, in such cases the edge effects will often be less sparse than under the true cladogram because a single causal mutation may now have to be represented by several non-zero 
(1) Construct the BT graph representation for the locus.

- $\quad$ Construct the cladogram for the locus under investigation using software such as PAUP [22].

- Compute the path between any pair of haplotypes in the cladogram.

(2) Fit an $L_{1}$-penalized (Lasso) CLR model in terms of the edge effects.

- $\quad$ Use $k$-CV to set the tuning parameter $\lambda$.

- $\quad$ Given the chosen $\lambda$, we get a model with a set of non-zero edge effects $S$.

(3) Refit the unpenalized CLR model using only the edge effects in $S$.

- $\quad$ Carry out an analysis of deviance test of this model against the global $H_{0}$ model.

- $\quad$ This produces a $\mathrm{p}$ value statistic, denoted by $p$.

(4) For $k=1,2, \ldots, N$, (e.g. $N=500$ is the number of permutations)

- Randomly flip the transmission status of the two haplotypes in each parent.

- $\quad$ For each permuted data set that so arises, repeat steps 2 and 3 and get a p value statistic, $p^{(k)}$.

Compute the significance level as the proportion of $p^{(k)} s$ that are less than $p$.

- $\quad$ Reject the global $H_{0}$ of no significant edge effects if the significance is $<\alpha$, for $\alpha=0.05$ for example.

edge effects under the inferred cladogram. Cross-validation provides a means to adjusting the amount of penalization accordingly.

\section{Assessing Significance through Permutation}

The $L_{1}$-penalized conditional logistic regression $\left(L_{1}\right.$-pCLR) provides a means for producing a model fit with sparse estimates of the edge effects. However, in the current context, our prime interest lies in testing the global $H_{0}$ that all edge effects are zero. Therefore, one needs a way to measure the statistical significance of the sparse model fit produced by $L_{1}$-pCLR. For this purpose, we propose the following procedure. First, let $S$ denote the set of edges with non-zero effects as determined by $L_{1}$-pCLR, along with $k$-CV. We then carry out a test of the global $H_{0}$ against the alternative $H_{1}: \gamma_{i_{1}, i_{2}}=0$ for all edges $\left\{i_{1}, i_{2}\right\}$ not in $S$. This can be done by refitting the unpenalized CLR (that is $\lambda=0$ ) under this restricted model and carrying out an analysis of deviance $\chi^{2}$ test of this model against $H_{0}$. (The degree of freedom is equal to the number of selected non-zero edge effects.) This produces a $\mathrm{p}$ value which we use as a statistic for measuring evidence against $H_{0}$. We note that this is a common strategy for evaluating the significance of Lasso-selected models. For example, it is adopted in the context of genome-wide association studies by $\mathrm{Wu}$ et al. [21].

This 'p value' statistic cannot be interpreted nominally as a significance level but needs to be adjusted to account for the model search. This can be achieved by constructing an appropriate permutation $H_{0}$ for this statistic with which a meaningful significance level can then be computed. Under the global $H_{0}$, each haplotype in a parent has $50 \%$ chance to be transmitted to the child. Therefore one can randomly permute the transmitted and untransmitted states of the two haplotypes within each parent with $50 / 50$ chance. For each permutation the whole process of fitting a $L_{1}$-pCLR and selecting $\lambda$ with $k$-CV can be carried out and the 'p value' statistic can be computed. After conducting a large number of such permutations, one can then pool the 'p value' statistics together and get a null distribution. The significance of the originally selected model can be computed as the proportion of times the permutation ' $p$ value' is smaller, and we can reject the global $H_{0}$ if, say, the significance level is less than $5 \%$.

A Sparse TDT for Haplotypes
Table 1. Haplotype structures and relative frequencies

\begin{tabular}{lllllll}
\hline Haplotype & \multicolumn{2}{l}{ Marker } & & \multicolumn{2}{c}{} & Frequency \\
\cline { 2 - 5 } & 1 & 2 & 3 & 4 & 5 & \\
\hline A & 1 & 1 & 1 & 1 & 1 & 0.180 \\
B & 0 & 0 & 1 & 1 & 1 & 0.214 \\
C & 1 & 1 & 0 & 1 & 0 & 0.194 \\
D & 0 & 0 & 1 & 0 & 1 & 0.100 \\
E & 1 & 1 & 0 & 1 & 1 & 0.277 \\
F & 0 & 1 & 0 & 1 & 1 & 0.006 \\
G & 0 & 0 & 0 & 1 & 1 & 0.014 \\
H & 1 & 1 & 1 & 0 & 1 & 0.006 \\
J & 1 & 1 & 0 & 0 & 1 & 0.004 \\
K & 0 & 1 & 1 & 1 & 1 & 0.006 \\
\hline
\end{tabular}

This example is from Hallman et al. [19] and was adopted in Seltman et al. [10]. The frequencies sum up to 1.001 and we normalize them in the computation.

From now on, we shall refer to the whole procedure of using $L_{1}$-pCLR with $k$-CV for model selection, and using permutation for evaluating the significance level as $L_{1}$-pCLR-based sparse TDT, or sparse TDT for short. This method is summarized in Box 1.

\section{Results}

In this section we carry out a power study of the proposed method through simulations and compare it to the original ET-TDT [10] to show the effect of incorporating sparsity on the edge effects. We again use the five-marker scenario adopted in Seltman et al. [10] as given in table 1 and figure 1 . We simulate the 10 haplotypes for a 
Table 2. Disease models for simulation

\begin{tabular}{lll}
\hline Model & Risk multiplier not equal to 1 & Type of disease-related mechanism \\
\hline NULL & none & no disease-related mutation event \\
\hline C & $\phi_{C}=1.4$ & \\
B & $\phi_{B}=1.4$ & \\
BD-eq & $\phi_{B}=\phi_{D}=1.3$ & \\
BD-neq & $\phi_{B}=1.1, \phi_{D}=1.4$ & \\
CE-eq & $\phi_{C}=\phi_{E}=1.3$ & \\
CE-neq & $\phi_{C}=1.4, \phi_{E}=1.1$ & \\
KBDG-eq & $\phi_{K}=\phi_{B}=\phi_{D}=\phi_{G}=1.25$ & single disease-related mutation \\
KBDG-neq & $\phi_{K}=1.1, \phi_{B}=1.2, \phi_{D}=\phi_{G}=1.3$ & \\
\hline CBG-eq & $\phi_{C}=\phi_{B}=\phi_{G}=1.3$ & two distinct disease-related mutations \\
CBG-neq & $\phi_{C}=1.3, \phi_{B}=1.4, \phi_{G}=1.5$ & D is associated with the disease \\
\hline DH-rec & $\phi_{D}=1.4, \phi_{H}=2$ & E and F are associated with the disease \\
EFG-rec & $\phi_{E}=1.3, \phi_{F}=1.8, \phi_{G}=1.8$ & G is a recombinant from B and E \\
BK-rec & $\phi_{B}=1.3, \phi_{K}=2$ & B is associated with the disease \\
& & K is a recombinant from A and B \\
\hline KBDG-clad & $\phi_{K}=1.2, \phi_{B}=1.3, \phi_{D}=1.1, \phi_{G}=1.4$ & incorrectly constructed cladogram - \\
& & D should be a child of A, not B \\
\hline
\end{tabular}

population of 100,000 pairs of parents for this five-marker locus according to the haplotype frequencies given in table 1. Given these parental haplotypes, we simulate the haplotypes for one child per parent-pair with equal transmission probability for the two haplotypes in each parent.

After generating the haplotypes, we simulate the disease status for the children according to 15 different disease models similar to the ones used in Seltman et al. [10]. (For ease of comparison, we adopt the same names for these models as in that paper.) These models were designed to imitate different mutational and recombinant scenarios that occurred during the evolutionary history of the locus, according to the underlying cladogram for the alleles (fig. 1). The models are presented in table 2 . In all of these models, the baseline disease risk for the child population is $p_{0}=0.2$. For a child with haplotypes $i$ and $j$ at the locus, where $i, j \in\{A, B, C, D, E, F, G, H, J, K\}$, the risk of disease $p_{i, j}$ takes a multiplicative form: $p_{i, j}=p_{0} \phi_{i} \phi_{j}$. Hereafter we refer to $\phi_{i}$ as the risk multiplier of haplotype $i$. The corresponding risk multipliers of the haplotypes for each of the 15 models are listed in the middle column of table 2. Haplotypes that do not contribute to the disease risk, i.e. whose risk multiplier is 1 , are omitted in the table. Note that although the models presented here are all multiplicative, our proposed method, being based completely on the conditional likelihood, does not depend on this particular aspect of the simulation.

For each of the models under consideration, after generating the disease status for the children population, we sample case-parent trios under three sample sizes $-\mathrm{n}=$ 500,750 , and 1,000 - and for each simulated data set we treat the haplotype phases as known and apply Method I: sparse TDT based on $L_{1}$-pCLR with 10 -fold CV for model selection and Method II: the original ET-TDT, which uses a Bonferroni-adjusted sequential testing procedure. For comparison, we apply two additional testing strategies spanning the two extremes of the sparsity spectrum - Method III: CLR with 'strong sparsity', which fits a sparse model with only one non-zero edge effect and Method IV: CLR with no sparsity imposed, i.e. $\lambda=0$. For Method III, the 'strong sparsity' test is carried out by fitting each of the $M-1$ models with a single non-zero edge effect and choose the most significant one as determined by the likelihood ratio test $\mathrm{p}$ value against $H_{0}$, and correct the significance level computed through the same permutation procedure as that for sparse TDT. For Method IV, we carry out a joint likelihood ratio test against the global $H_{0}$ that all edge effects are nil with no constraints under $H_{1}$.

The simulation is repeated 500 times for each model/ sample size combination. Each time, 500 permutations 
are used to evaluate the significance levels. We note that since Method IV is based on a single global hypothesis test for the entire locus, its $p$ value can be used without permutation correction if a single locus is under consideration. (We will discuss strategies for genome-wide studies in the Discussion section.) The power is estimated by the fraction of times $H_{0}$ is rejected at the $5 \%$ level. The power at the 5\% level (vs. sample size) of the four methods is presented in figure 2. Note that the power plot for the $\mathrm{H}_{0}$ model and table 3 show that in the current example both our permutation procedure and the ET-TDT are able to control the type 1 error rate. Overall, taking sparsity into account pays off in the power - the sparse TDT consistently outperforms the ET-TDT. In fact it performs the best for all simulated models except EFG-rec. The good performance of the joint testing approach (labeled as 'no sparsity joint' in fig. 2) for EFG-rec is expected - for that model there are five non-zero edge effects out of a total of nine edges. The performance of the joint testing approach and that of the 'strong sparsity' approach, however, are highly variable and sensitive to the particular underlying disease model. This is not surprising as neither of these approaches is adaptive to the actual sparsity in the underlying model.

\section{Performance under a Larger Cladogram}

Our proposed approach is based on the idea that the non-zero edge effects are sparse. As a result, one may expect that the gain in performance will increase as the underlying sparsity increases. To investigate this, we extend the original cladogram to include four more haplotypes - $L, M, N, O$ - by adding four more markers into the locus. The corresponding haplotype structures in terms of the marker alleles are given in table 4 . The extended cladogram is given in figure 3.

The same simulation procedure under the 15 disease models is repeated for this extended set of haplotypes, except that now we consider larger sample sizes $-\mathrm{n}=500$, 1,000 and 1,500. The power versus sample size for the four methods is presented in figure 4 and the type 1 errors are given in table 5. Several interesting observations can be made. First, the 'no sparsity' joint testing approach performs better than the original ET-TDT under every model under this extended cladogram. This suggests that the stringent Bonferroni approach to correct for multiple testing adopted by the ET-TDT may be overly conservative when the number of haplotypes is large. Second, the overall performance of the 'strong sparsity' approach, in comparison to the other methods, is better in this more sparse cladogram, as one would expect. Finally, the sparse
Table 3. Type 1 errors for the four methods by sample size

\begin{tabular}{llll}
\hline Method & \multicolumn{3}{l}{ Sample size } \\
\cline { 2 - 4 } & 500 & 750 & 1,000 \\
\hline$L_{1}$-pCLR with CV & $4.3 \%$ & $3.5 \%$ & $2.4 \%$ \\
ET-TDT & $4.9 \%$ & $4.8 \%$ & $5.8 \%$ \\
Strong sparsity & $3.3 \%$ & $2.6 \%$ & $2.7 \%$ \\
No sparsity & $3.8 \%$ & $3.1 \%$ & $3.0 \%$ \\
\hline
\end{tabular}

Table 4. Extended haplotype structure and their frequencies

\begin{tabular}{lllllllllll}
\hline \multirow{2}{*}{ Haplotype } & \multicolumn{1}{l}{ Marker } & \multicolumn{1}{l}{ Frequency } \\
\cline { 2 - 7 } & 1 & 2 & 3 & 4 & 5 & 6 & 7 & 8 & 9 & \\
\hline $\mathrm{A}$ & 1 & 1 & 1 & 1 & 1 & 1 & 1 & 1 & 1 & 0.128 \\
$\mathrm{~B}$ & 0 & 0 & 1 & 1 & 1 & 1 & 1 & 1 & 1 & 0.153 \\
$\mathrm{C}$ & 1 & 1 & 0 & 1 & 0 & 1 & 1 & 1 & 1 & 0.138 \\
$\mathrm{D}$ & 0 & 0 & 1 & 0 & 1 & 1 & 1 & 1 & 1 & 0.071 \\
$\mathrm{E}$ & 1 & 1 & 0 & 1 & 1 & 1 & 1 & 1 & 1 & 0.198 \\
$\mathrm{~F}$ & 0 & 1 & 0 & 1 & 1 & 1 & 1 & 1 & 1 & 0.004 \\
$\mathrm{G}$ & 0 & 0 & 0 & 1 & 1 & 1 & 1 & 1 & 1 & 0.010 \\
$\mathrm{H}$ & 1 & 1 & 1 & 0 & 1 & 1 & 1 & 1 & 1 & 0.004 \\
$\mathrm{~J}$ & 1 & 1 & 0 & 0 & 1 & 1 & 1 & 1 & 1 & 0.003 \\
$\mathrm{~K}$ & 0 & 1 & 1 & 1 & 1 & 1 & 1 & 1 & 1 & 0.004 \\
$\mathrm{~L}$ & 1 & 1 & 1 & 1 & 1 & 1 & 1 & 1 & 0 & 0.107 \\
$\mathrm{M}$ & 1 & 1 & 1 & 1 & 1 & 0 & 1 & 1 & 0 & 0.086 \\
$\mathrm{~N}$ & 0 & 1 & 1 & 1 & 1 & 1 & 1 & 0 & 1 & 0.057 \\
$\mathrm{O}$ & 1 & 1 & 0 & 0 & 1 & 1 & 0 & 1 & 1 & 0.036 \\
\hline
\end{tabular}

TDT is again the best performing method overall - the gain in power over the original ET-TDT is substantial when the underlying model is indeed very sparse. At the same time, for less sparse models such as EFG-rec and BKrec (each of them has 5 non-zero edge effects under the extended cladogram) jointly testing all the effects actually performed better. While this phenomenon is something one would expect, it demonstrates itself more clearly in the extended cladogram than in the original. This is not because of the increased size of the cladogram - in fact one would expect the joint testing approach to be even more powerful in smaller cladograms where non-zero edge effects constitute a larger proportion of all edge effects. Instead, this is probably due to a combination of two reasons: (1) the frequencies of the disease-related haplotypes have decreased in the simulation for the extended cladogram, and (2) under the extended cladogram, the $\mathrm{BD}$-rec model has one extra non-zero edge effect. 


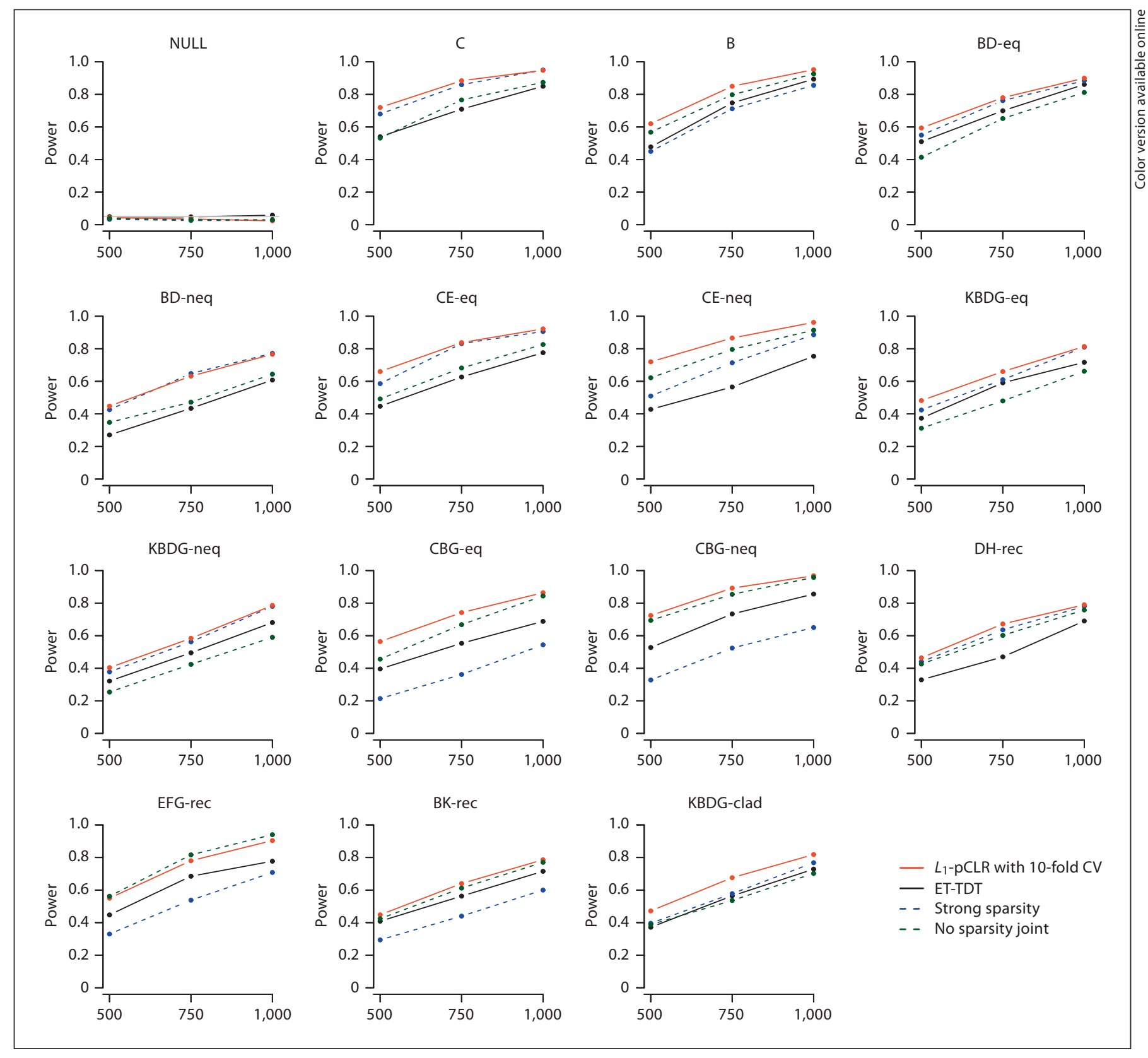

Fig. 2. Power at the $5 \%$ level versus sample size under the 15 models for four methods: (1) red (grey in the printed version) solid represents sparse TDT based on $L_{1}$-pCLR with 10-fold CV; (2) black solid represents ET-TDT; (3) blue (black in the printed version) dashed represents CLR with 'strong sparsity', and (4) green (grey in the printed version) dashed represents joint testing using CLR with no sparsity.

\section{Discussion}

In this work we have proposed an extension to the ETTDT for testing haplotype transmission disequilibrium in case-parent trios. Like the original ET-TDT, our proposed method utilizes evolutionary information of the locus through the corresponding cladogram. The original ET-TDT uses the cladogram as a guide for constructing hypotheses and thereby reducing the number of tests, while we, in addition, use the cladogram as a way to exploit sparsity in mutational events. In particular, we have introduced the notion of a $\mathrm{BT}$ graph by modeling the 
transmission event of each parent conditional on their haplotypes with a BT model parameterized in terms of the edges in the underlying cladogram. This allows us to impose sparsity over these edge effects by simply introducing an $L_{1}$ penalty term into the conditional likelihood. The motivation for imposing sparsity on the edge effects is that the causal mutations for diseases are rare, and so each susceptibility locus is expected to contain at most a small number of them.

One may consider imposing sparsity directly on the haplotype effects instead of the edge effects. For example, one can impose sparsity on the difference between the effect of the most common haplotype and those of the other ones. In doing so, one eliminates the need for constructing the cladogram, but at the same time does not utilize any evolutionary information. This is undesirable because even a single mutational event in the evolutionary history could lead to a large number of haplotypes with non-zero effects. (For example, consider the model KBDG-eq in our power study.) In our framework, evolutionary information is utilized through the assumption that a small number of causal mutations may be embedded in a small number of edge effects.

As a reviewer pointed out, there can be other ways to imposing sparsity into the hypothesis tests carried out by the ET-TDT without using the BT graph representation. In this regard, we believe that the BT graph is a particularly appealing tool for achieving this goal mainly for two reasons. First, the reparameterization of the allelic association into a collection of edge effects allows us to use simple, standard penalized regression tools and software to carry out inference. In turn, this also greatly simplifies the multiple testing correction procedure as each cladogram is tested jointly under this framework.

While we have developed our method in the context of testing a single locus, the framework can be applied in genome-wide or candidate gene studies, where there are often a large number of genetic loci to test for potential linkage and association. One conceptually easy way to carry out the test over multiple loci is to use the significance level of each locus computed through locus-by-locus permutation. For example, one can simply apply to these locus-specific permutation $\mathrm{p}$ values a standard multiple testing adjustment such as a Bonferroni correction. However, in situations where the number of loci is large, the adjusted significance threshold of nominal $\mathrm{p}$ values can be very small, e.g. $<10^{-5}$, and therefore it can require a prohibitively large number of permutations to estimate $p$ values up to this level of precision for all the loci. In this case, a useful strategy is to proceed in mul-

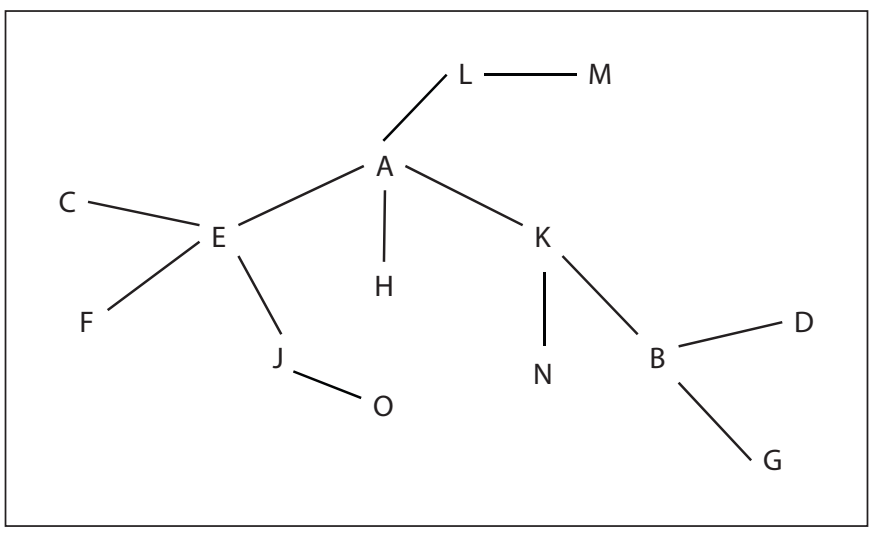

Fig. 3. Extended cladogram. Four additional haplotypes $L, M, N$, and $O$ are added.

Table 5. Type 1 errors of the four methods by sample size for the extended cladogram

\begin{tabular}{llll}
\hline Method & \multicolumn{3}{l}{ Sample size } \\
\cline { 2 - 4 } & 500 & 1,000 & 1,500 \\
\hline$L_{1}$-pCLR with CV & $3.6 \%$ & $5.8 \%$ & $4.6 \%$ \\
ET-TDT & $2.8 \%$ & $5.0 \%$ & $5.3 \%$ \\
Strong sparsity & $5.4 \%$ & $5.0 \%$ & $5.0 \%$ \\
No sparsity & $6.0 \%$ & $6.2 \%$ & $4.6 \%$ \\
\hline
\end{tabular}

tiple stages. For example, one can start by running 1,000 permutations for each locus and then find those that are significant at the $1 \%$ level, and run 100,000 permutations for each of these and find those that are significant at $0.01 \%$, and so on and so forth.

An alternative strategy is to construct an appropriate permutation $H_{0}$ distribution for the nominal significance level of the most significant sparse model over all candidate loci, denoted by $p_{\text {min }}^{L_{1}}$. In designing the appropriate permutation procedure, if one's goal is to imitate as closely as possible the underlying biological process, one should ideally maintain the linkage structure across the loci - the more linked two loci are with each other, the more likely they should be permuted together. A randomization procedure like this will break down the linkage and the linkage disequilibrium between the loci and the disease status, as is desired in building $H_{0}$. The exact manner in which such randomization should be carried out is interesting but beyond the scope of this paper. In the current context, however, there is a simple 'proxy' to 


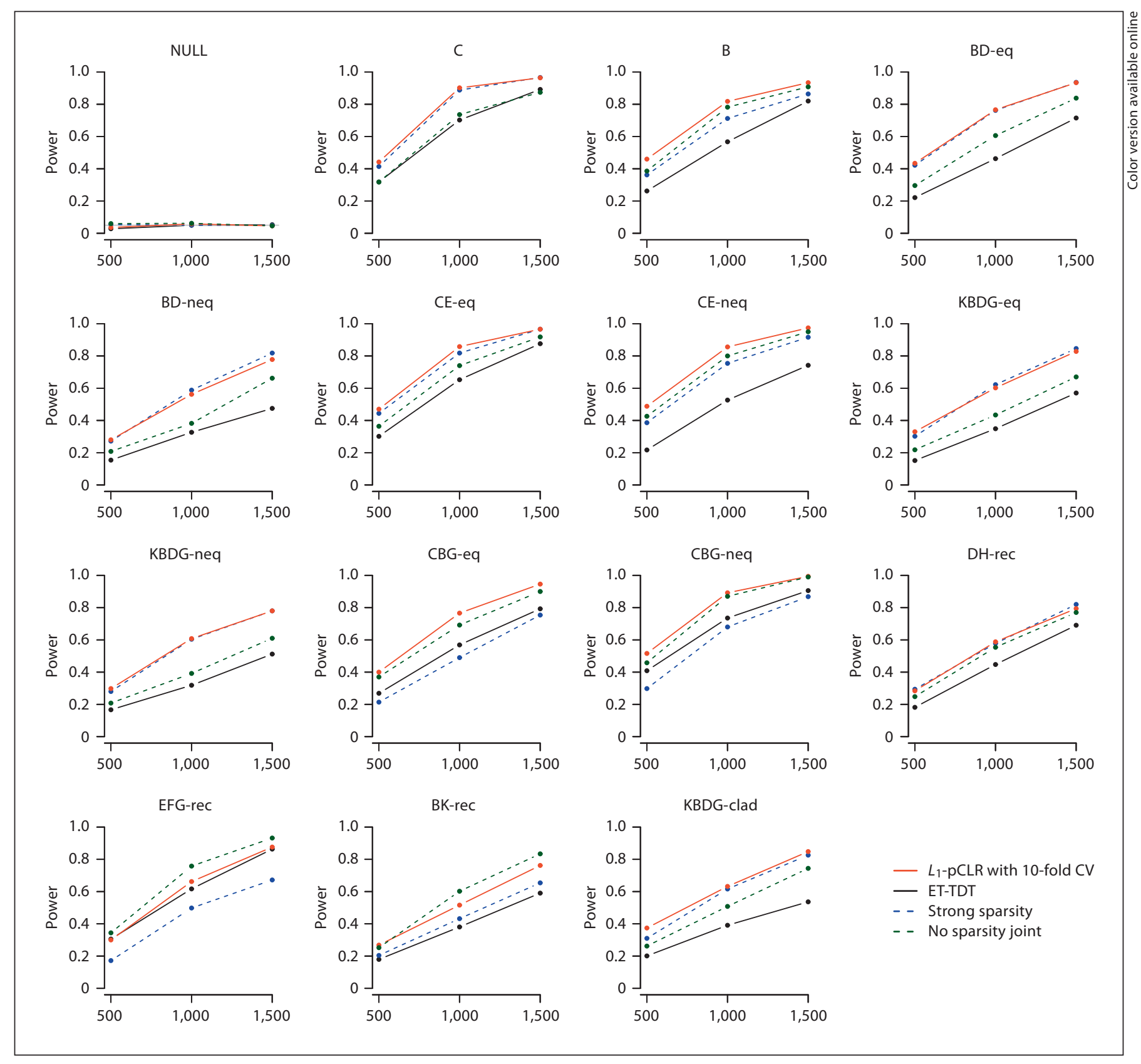

Fig. 4. Power at the $5 \%$ level versus sample size for the extended cladogram under the 15 models for four methods: (1) red (grey in the printed version) solid represents sparse TDT based on $L_{1}$-pCLR with 10-fold CV; (2) black solid represents ET-TDT; (3) blue (black in the printed version) dashed represents CLR with 'strong sparsity', and (4) green (grey in the printed version) dashed represents joint testing using CLR with no sparsity.

this procedure. That is, to permute the transmission states at all loci for each parent together. In other words, for each parent we flip a coin, and if the coin shows heads, we swap the transmitted and the untransmitted haplotypes at all loci for that parent. If it shows tail, we leave the transmission states of the haplotypes unchanged at all loci for that parent. For each such permutation we recompute $p_{\text {min }}^{L_{1}}$. The permutation $H_{0}$ distribution we get by pooling these values can then be used for calculating genome-wide $\mathrm{p}$ values. This simplified permutation proce- 
dure does not imitate the actual linkage among the markers, but it does break down both the linkage and the linkage disequilibrium between the loci and the disease status, while maintaining the linkage disequilibrium among the markers.

Our method is designed based on the assumption that there is no ambiguity in determining what haplotype is transmitted to the child from each parent. As the density of genetic markers gets higher, the problem of ambiguous haplotype phase will occur less and less frequently. Nevertheless, simple strategies to deal with ambiguous haplotype phase include imputing the phase with the most likely allele [13]. As mentioned at the beginning of this work, experimental technologies are now available that allow us to attain phased genotype information from the subjects directly [1], eliminating the phase problem com- pletely. While the development of such technology is still in its early stage and so the cost is relatively high at the time of this writing, we expect their use in the future to become more prevalent.

\section{Acknowledgments}

The authors want to thank two anonymous referees and the editor for very helpful comments. W.H.W. is supported in part by NIH grants R01HG004634 and R01HG005717, and NSF grant DMS-0906044. A.B.O. is supported by NSF-DMS0906056. Much of the computation in this work was carried out on computer resources supported by NSF awards CNS-0619926 and DMS0821823 . The computation is done in the statistical sofware R using packages 'igraph' [23] and 'penalized' [24].

\section{References}

1 Yang $\mathrm{H}$, Chen X, Wong WH: Completely phased genome sequencing through chromosome sorting. P Natl Acad Sci USA 2011; 108:12-17.

2 Falk CT, Rubinstein P: Haplotype relative risks: an easy reliable way to construct a proper control sample for risk calculations. Ann Hum Genet 1987;51:227-233.

3 Ott J: Statistical properties of the haplotype relative risk. Genet Epidemiol 1989;6:127130.

4 Spielman RS, McGinnis RE, Ewens WJ: Transmission test for linkage disequilibrium: the insulin gene region and insulin-dependent diabetes mellitus (iddm). Am J Hum Genet 1993;52:506.

5 Ewens WJ, Spielman RS: The transmission/ disequilibrium test: history, subdivision, and admixture. Am J Hum Genet 1995;57: 455.

-6 Spielman RS, Ewens WJ: The TDT and other family-based tests for linkage disequilibrium and association. Am J Hum Genet 1996; 59:983.

7 Lazzeroni L, Lange K: A conditional inference framework for extending the transmission/disequilibrium test. Hum Hered 1998; 48:67-81.

8 Clayton D: A generalization of the transmission/disequilibrium test for uncertain-haplotype transmission. Am J Hum Genet 1999; 4:1170-1177.

9 Clayton D, Jones H: Transmission/disequilibrium tests for extended marker haplotypes. Am J Hum Genet 1999;65:1161-1169.
Seltman H, Roeder K, Devlin B: Transmission/disequilibrium test meets measured haplotype analysis: family-based association analysis guided by evolution of haplotypes. Am J Hum Genet 2001;68:1250-1263.

11 Rabinowitz D, Laird N: A unified approach to adjusting association tests for population admixture with arbitrary pedigree structure and arbitrary missing marker information. Hum Hered 2000;50:211-223.

-12 Zhao H, Zhang S, Merikangas KR, Trixler M, Wildenauer DB, Sun F, Kidd KK: Transmission/disequilibrium tests using multiple tightly linked markers. Am J Hum Genet 2000;67:936

13 Zhang S, Sha Q, Chen HS, Dong J, Jiang R: Transmission/disequilibrium test based on haplotype sharing for tightly linked markers. Am J Hum Genet 2003;73:566-579.

14 Templeton AR, Boerwinkle E, Sing CF: A cladistic analysis of phenotypic associations with haplotypes inferred from restriction endonuclease mapping. I. Basic theory and an analysis of alcohol dehydrogenase activity in drosophila. Genetics 1987;117:343.

15 Templeton AR, Sing CF, Kessling A, Humphries S: A cladistic analysis of phenotype associations with haplotypes inferred from restriction endonuclease mapping. II. The analysis of natural populations. Genetics 1988;120:1145.

16 Templeton AR, Crandall KA, Sing CF: A cladistic analysis of phenotypic associations with haplotypes inferred from restriction endonuclease mapping and DNA sequence data. III. Cladogram estimation. Genetics 1992;132:619.
17 Templeton A, Sing CF: A cladistic analysis of phenotypic associations with haplotypes inferred from restriction endonuclease mapping. IV. Nested analyses with cladogram uncertainty and recombination. Genetics 1993; 134:659.

18 Templeton AR: A cladistic analysis of phenotypic associations with haplotypes inferred from restriction endonuclease mapping or DNA sequencing. V. Analysis of case/control sampling designs: Alzheimer's disease and the apoprotein e locus. Genetics 1995;140: 403.

19 Hallman DM, Visvikis S, Steinmetz J, Boerwinkle E: The effect of variation in the apolipoprotein $B$ gene on plasma lipid and apolipoprotein B levels. I. A likelihood-based approach to cladistic analysis. Ann Hum Genet 1994;58:35-64.

20 Tibshirani R: Regression shrinkage and selection via the Lasso. J Royal Stat Soc B 1996; 58:267-288.

21 Wu TT, Chen YF, Hastie T, Sobel E, Lange K: Genome-wide association analysis by Lasso penalized logistic regression. Bioinformatics 2009;25:714-721.

22 Swofford DL: PAUP* Phylogenetic Analysis Using Parsimony (*and Other Methods), Version 4.04beta. Sinauer Associates, Sunderland, 2003.

23 Csardi G, Nepusz T: The igraph software package for complex network research. InterJournal, Complex Systems 1695, 2006.

24 Goeman JJ: L1 penalized estimation in the Cox proportional hazards model. Biom J 2010;52:70-84. 